\title{
Charge-magnetic interference resonant scattering studies of ferromagnetic crystals and thin films
}

\author{
D. Haskel ${ }^{1, a}$, E. Kravtsov ${ }^{1,2}$, Y. Choi ${ }^{1}$, J.C. Lang ${ }^{1}$, Z. Islam ${ }^{1}$, G. Srajer ${ }^{1}$, \\ J.S. Jiang ${ }^{3}$, S.D. Bader ${ }^{3}$, and P.C. Canfield ${ }^{4}$ \\ 1 Advanced Photon Source, Argonne National Laboratory, USA \\ 2 Institute of Metal Physics, Russian Academy of Sciences, and Ural Federal University, \\ Ekaterinburg, Russia \\ ${ }^{3}$ Materials Science Division, Argonne National Laboratory, USA \\ 4 Department of Physics and Ames Laboratory, Iowa State University, USA
}

Received 19 December 2011 / Received in final form 23 March 2012

Published online 15 June 2012

\begin{abstract}
The element- and site-specificity of X-ray resonant magnetic scattering (XRMS) makes it an ideal tool for furthering our understanding of complex magnetic systems. In the hard X-rays, XRMS is readily applied to most antiferromagnets where the relatively weak resonant magnetic scattering $\left(10^{-2}-10^{-6} I_{c}\right)$ is separated in reciprocal space from the stronger, Bragg charge scattered intensity, $I_{c}$. In ferro(ferri)magnetic materials, however, such separation does not occur and measurements of resonant magnetic scattering in the presence of strong charge scattering are quite challenging. We discuss the use of charge-magnetic interference resonant scattering for studies of ferromagnetic (FM) crystals and layered films. We review the challenges and opportunities afforded by this approach, particularly when using circularly polarized X-rays. We illustrate current capabilities at the Advanced Photon Source with studies aimed at probing site-specific magnetism in ferromagnetic crystals, and interfacial magnetism in films.
\end{abstract}

\section{Introduction}

Ferromagnetic (FM) materials in bulk- and thin-film form are central components of a wide variety of commercial technologies including car starters, alternators for wind power generation, media and read-heads of computer hard-drives, and more. The quest for ever-smaller magnetic devices, and a related need for achieving increased density of magnetic elements and stored energy, are driving a surge in the complexity of artificial FM structures now synthesized in both 3D and 2D form. The quest for novel bulk materials for energy applications, such as stronger permanent magnets or efficient magnetocaloric materials, more often than not results in complex crystal structures with large unit cells. Multiple inequivalent crystal sites within the unit cell may be occupied by magnetic elements of the same specie. Similar complexity is

a e-mail: haskel@aps.anl.gov 
found in magnetic nanostructures where FM elements meet other magnetic, or nonmagnetic, elements at interfaces to form artificial periodic structures with in-plane or out-of-plane periodicities. This evolution in the complexity of magnetic materials calls for novel, element- and site-specific characterization tools, an area where $\mathrm{X}$-ray resonant scattering techniques have much to offer. In particular, X-ray resonant magnetic scattering (XRMS) [1-3] has evolved into a unique probe of complex bulk and film magnetic systems where the energy $(E)$, scattering-vector $(\boldsymbol{Q})$ and incoming/outgoing polarization $\left(\hat{\epsilon}, \hat{\epsilon}^{\prime}\right)$ dependence of the resonant scattering process can be exploited to probe magnetic ordering and magnetic moment orientation with element- and site-specificity. While neutron scattering remains the natural choice for the investigation of magnetic structures due to the strong interaction of neutrons with both the nuclear potential and the electronic magnetic moments, the ability to preferentially "turn on/off" magnetic scattering from selected species in XRMS is a definitive advantage in complex systems. (Here we are neglecting the much weaker, non-resonant magnetic scattering, $10^{-7}-10^{-8} I_{c}$ ) [4-6]. In the case of thin films probed by XRMS in specular reflection geometry (also known as magnetic reflectivity), the higher brilliance of synchrotron radiation sources relative to that of neutron sources enables measurement of the rapidly decaying $\left(Q^{-4}\right)$ magnetic reflectivity to higher $\boldsymbol{Q}$ vectors, which oftentimes translates into a $\times(5-10)$ gain in the spatial resolution of refined magnetization profiles [7].

While antiferromagnetic (AFM) structures, whether commensurate or incommensurate with the lattice, display a different periodicity than the chemical lattice (doubled for collinear, commensurate AFM structures), the periodicities of magnetic and chemical lattices are the same in ferro(ferri)-magnetic structures. The corresponding charge and magnetic scattering overlap in reciprocal space, and isolation of the much weaker resonant magnetic scattering is challenging, as discussed later below. The spatial overlap of charge and magnetic scattering, however, allows for possible interference between these two scattering channels when their outgoing polarization states are not orthogonal to one another [8]. This paper focuses on describing the conditions under which this interference process takes place, and how this charge-magnetic interference scattering can be exploited for quantitative studies of ferromagnetic crystals and layered magnetic films.

\section{What does it take to isolate charge and resonant magnetic scattering in ferromagnets?}

The electric dipole $(E 1)$ scattering amplitude for a single magnetic ion $n$ near resonance is given by

$$
f_{n}(Q, E)=\left[f_{0, n}(Q)+f_{c, n}(E)\right]\left(\hat{\epsilon}^{\prime *} \cdot \hat{\epsilon}\right)-i f_{m, n}(E)\left(\hat{\epsilon}^{*} \times \hat{\epsilon}\right) \cdot \hat{m}_{n},
$$

where $f_{0}, f_{c}, f_{m}$ are non-resonant (Thomson), resonant charge, and resonant magnetic scattering amplitudes, respectively, with the last two being complex quantities responsible for anomalous dispersion [1-3]. Polarization-dependent terms contain incoming and outgoing polarization vectors $\left(\hat{\epsilon}, \hat{\epsilon}^{\prime}\right)$, complex for circular polarization, and $\hat{m}$, the local magnetic moment direction. Note that we neglect here weaker nonresonant magnetic scattering and resonant magnetic scattering that is quadratic in the moment direction $[1-3,6]$.

In a lattice of magnetic ions, the scattered intensity is given by the modulus square of the structure factor, $|F(Q, E)|^{2}=\left|\sum_{n} f_{n} e^{i \boldsymbol{Q} \cdot \boldsymbol{r}_{n}}\right|^{2}$ where the sum is over all scattering atoms in the unit cell, with $f_{n}$ given in Eq. (1). Note that here, as in 


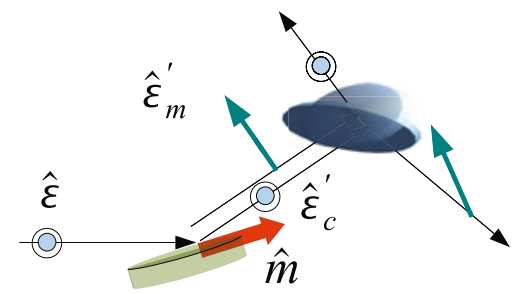

Fig. 1. Orthogonal outgoing polarization states in charge and magnetic scattering channels prevents C-M interference scattering from taking place. While a polarization analyzer (second crystal) can be used to separate charge and magnetic scattering, practical suppression rates are not enough to achieve such separation in most experiments on ferromagnets at hard X-ray resonances.

the remainder of the paper, we use scalar anomalous scattering factors corresponding to isotropic scattering, i.e., independent of polarization. A tensorial description of resonant scattering factors is needed for anisotropic scattering, commonly found in resonant soft X-ray scattering experiments where resonant transitions at the $L_{2,3}$ and $\mathrm{M}_{4,5}$ edges of transition metal ions and rare-earths, respectively, probe localized $3 d$, $4 f$ orbitals. This is mitigated in the hard X-rays where the more "diffuse" (delocalized) $4 p, 5 d$ states are probed at their $K$ and $L_{2,3}$ edges. However, a proper treatment of the polarization-dependence of scattering factors ought to be included in quantitative analysis of C-M interference data obtained in single-crystalline samples with symmetry lower than cubic $[9,10]$. In ferro(ferri)-magnets, charge and magnetic terms in Eq. (1) contribute to the structure factor at the same $\boldsymbol{Q}$ vector (same periodicity hence same phase term) so the squaring of the structure factor would in principle contain pure charge, pure magnetic, and charge-magnetic cross-terms (C-M interference terms). However, while overlapping charge and magnetic contributions in reciprocal space is a necessary condition for C-M interference to take place, this interference also requires non-orthogonal polarization states of charge- and magnetically-scattered outgoing photons (i.e., $\hat{\epsilon}_{c}^{\prime} \cdot \hat{\epsilon}_{m}^{\prime} \neq 0$ ).

Because of the different polarization dependence of charge and resonant magnetic scattering terms in Eq. (1) one may be able to separate charge and resonant magnetic scattering by analyzing the polarization of the outgoing (scattered) $\mathrm{X}$-ray beam [8]. Consider a vertical scattering plane and a horizontally polarized incident X-ray beam, as delivered by a linear undulator or bending magnet at a synchrotron source, and a magnetic moment direction in the scattering plane (Fig. 1). [We use the $(\hat{\sigma}, \hat{\pi})$ representation to describe polarization components perpendicular and parallel to the scattering plane, respectively. So here $\hat{\epsilon}=\hat{\sigma}]$. A non-zero, charge scattered intensity requires an unrotated outgoing polarization $\left(\hat{\epsilon}_{c}^{\prime}=\hat{\sigma}^{\prime}\right)$, while in this geometry non-zero resonant magnetic scattering requires a rotated outgoing polarization $\left(\hat{\epsilon}_{m}^{\prime}=\hat{\pi}^{\prime}\right.$ ), see Eq. (1). (Fig. 1 shows charge and magnetic scattering channels with separate lines for clarity.) The orthogonal polarizations of charge- and magnetically-scattered beams prevents any C-M interference from taking place, and the scattered intensity contains only pure charge and magnetic scattering terms, $I(Q, E)=\left|\sum_{n} f_{e, n}(Q, E) e^{i \boldsymbol{Q} \cdot \boldsymbol{r}_{n}}\right|^{2}+\left|i \sum_{n} f_{m, n}(E)\left(\hat{k}_{f} \cdot \hat{m}_{n}\right) e^{i \boldsymbol{Q} \cdot \boldsymbol{r}_{n}}\right|^{2}$, where non-resonant and resonant charge scattering amplitudes were merged into $f_{e}$ and $\left(\hat{\epsilon}_{m}^{\prime} \times \hat{\epsilon}\right)=\left(\hat{\pi}^{\prime} \times \hat{\sigma}\right)=\hat{k}_{f}$ was used, $\hat{k}_{f}$ being the direction of the scattered beam. For resonances in the hard X-ray region (e.g. $L_{2,3}$ edges of rare-earths, $5 d$ elements, and actinides, or $K$-edges of transition metals), $\left|f_{m} / f_{e}\right|^{2}$ is usually in the $10^{-4}-10^{-6}$ range (the exception is the $M_{4,5}$ edges of actinides and $L_{2,3}$ edges of $4 d$ elements where the resonant magnetic scattering is more intense [11-14]). Therefore, a measurement of 


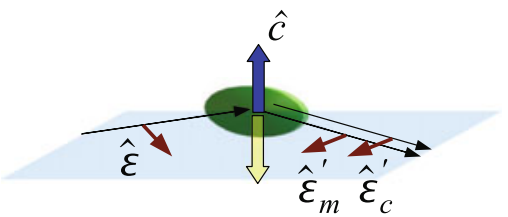

Fig. 2. Differential measurement of total scattered intensity for opposite magnetization direction (without polarization analysis) yields C-M interference scattering. The flipping ratio is largely enhanced for scattering angles near $90^{\circ}$ where the pure charge scattering is suppressed.

scattered intensity with no polarization analysis of the scattered beam is basically a measurement of the charge scattered intensity.

Since the outgoing polarizations of charge and magnetic channels are orthogonal, one could try to separate these two contributions with a polarization analyzer; i.e., a non-magnetic crystal with a Bragg reflection satisfying $2 \theta_{B}=90^{\circ}$ at the resonant energy. Due to the polarization dependence of Thomson scattering in the analyzer crystal $\left(\hat{\epsilon}_{c}^{\prime} \cdot \hat{\epsilon}_{c}\right)$, the magnetic scattering will be detected when the analyzer is oriented to diffract in the horizontal plane, while the charge scattering (from the sample) will be detected when the analyzer is oriented to diffract in the vertical plane, as schematically shown in Fig. 1. However, an exact Bragg condition in the analyzer needed for perfect suppression of the orthogonal polarization component is hard to achieve. What degree of suppression is needed? Based on the values of $\left|f_{m} / f_{e}\right|^{2}$, a polarization suppression of at least $10^{4}$ is typically needed. As an example, the polarization suppression for $2 \theta_{B}=89^{\circ}$ is $\approx 3,300\left(\cos ^{2} 2 \theta_{\mathrm{B}}\right)^{-1}$ while that for $2 \theta_{B}=88^{\circ}$ is only $\approx 820$, far from the values required for isolation of magnetic scattering in most cases. (Typical polarization suppression achievable in standard experiments, also dictated by the finite solid angle sustained by the detector and crystal analyzer's mosaicity, is $\approx$ 100.) An alternative approach to the difficult task of separating charge and magnetic scattering contributions in ferro(ferri)-magnets is to construct a scattering geometry where they do interfere, and detect this stronger interference scattering $\left(10^{-2}-10^{-3} I_{c}\right)$ by subtracting the pure charge scattering contribution in a differential measurement.

\section{Charge-magnetic interference scattering}

\subsection{Linear polarization}

Consider a horizontal scattering plane, a linearly polarized X-ray beam $(\hat{\epsilon}=\hat{\pi})$, and a magnetization oriented in the vertical direction (i.e. normal to the scattering plane), as shown in Fig. 2. In this geometry the outgoing polarization in both charge and magnetic scattering channels remains unrotated. (If $\hat{\epsilon}^{\prime}=\hat{\sigma}^{\prime}$, then $\left(\hat{\epsilon}^{\prime} \cdot \hat{\epsilon}\right)=\left(\hat{\epsilon}^{\prime} \times \hat{\epsilon}\right)$ $\cdot \hat{m}=0)$. Non-orthogonal, outgoing polarization states lead to C-M interference scattering in addition to pure charge and magnetic scattering. For magnetization oriented up $(\uparrow)$ or down $(\downarrow)$ we have:

$$
\begin{aligned}
I^{\uparrow \downarrow}(Q, E)= & \left|\sum_{n} f_{e, n}(Q, E) \cos 2 \theta e^{i \boldsymbol{Q} \cdot \boldsymbol{r}_{n}}\right|^{2}+\left|i \sum_{n} f_{m, n}(E) \sin 2 \theta e^{i \boldsymbol{Q} \cdot \boldsymbol{r}_{n}}\right|^{2} \\
& \mp i \sum_{n, l} f_{e, n}^{*} f_{m, l} \cos 2 \theta \sin 2 \theta e^{i \boldsymbol{Q} \cdot\left(\boldsymbol{r}_{l}-\boldsymbol{r}_{n}\right)}+c . c .
\end{aligned}
$$

since the pure charge and magnetic scattering terms are insensitive to the up/down orientation of the magnetization while the $\mathrm{C}-\mathrm{M}$ interference term changes sign upon 
reversal of magnetization direction. Hence, a differential measurement of total scattered intensity (without polarization analysis) for up and down directions of magnetization yelds $I^{\uparrow}-I^{\downarrow}=-2 i \sum_{n, l} f_{e, n}^{*} f_{m, l} \cos 2 \theta \sin 2 \theta e^{i \boldsymbol{Q} \cdot\left(\boldsymbol{r}_{l}-\boldsymbol{r}_{n}\right)}+$ c.c., i.e., the differential measurement gets rid of the strong, pure charge scattering. Summing these scattered intensities yields $I^{\uparrow}+I^{\downarrow}=2\left|\sum_{n} f_{e, n}(Q, E) \cos 2 \theta e^{i \boldsymbol{Q} \cdot \boldsymbol{r}_{n}}\right|^{2}$ since the pure magnetic scattering can be neglected (this may not be the case for soft X-ray resonances).

Since the ratio of C-M interference to pure charge scattering terms for TM $K$-edges and RE $L$-edges is of the order of $f_{m} / f_{e} \approx 10^{-2}-10^{-3}$ (neglecting structure factor and polarization effects) the measurement of total scattered intensity has to be carried out with a precision in the $0.01-0.1 \%$ range for an accurate subtraction of C-M interference scattering in a differential measurement (statistical error achieved with $10^{6}-10^{8}$ photons in the scattered beam). This is readily achieved with a high countrate detector, such as an avalanche photodiode, since the measurements are usually performed on strong (allowed) Bragg reflections from single crystals, or strong specular reflections from thin films. A flipping ratio is defined as FR $=I^{\uparrow}-I^{\downarrow} / I^{\uparrow}+I^{\downarrow}$. The flipping ratio is a much more robust quantitity to determine experimentally, especially when doing energy scans across a resonance at fix $\boldsymbol{Q}$, or for $\boldsymbol{Q}$ scans at a fixed resonant energy. Unlike the bare difference intensity, the flipping ratio is quite robust to sample misalignment away from perfect Bragg or specular condition, so long as the deviation is safely within the characteristic rocking curves.

Note that the polarization dependent terms yield a tan $2 \theta$ dependence of the FR on scattering angle $2 \theta[15,16]$. This quantitiy diverges as the scattering angle approaches $90^{\circ}$ (charge scattering $\rightarrow 0$ ). Hence, the FR signal is hugely enhanced for Bragg reflections with $\theta_{B} \sim 45^{\circ}$ (or in the soft X-rays for specular reflection at $45^{\circ}$ incidence angle). Under those conditions the signal-to-noise requirements are largely relaxed, a great advantage of this geometry [17]. In the absence of this condition, it is important to carry out the differential measurement at each energy point in resonant energy scans at fix $\boldsymbol{Q}$, and at each $\boldsymbol{Q}$ point in $\boldsymbol{Q}$ scans at fix energy.

Since a superconducting magnet may be needed to switch the magnetization, requiring long switching times of minutes during which beam conditions/alignment may change, the need to switch magnetization direction is a disadvantage compared to the polarization switching scheme discussed in Sect. 3.2. Furthermore, since magnetization switching is needed for a measurement of the FR, field-dependent studies are not readily pursued in this geometry. We note that the first resonant magnetic scattering experiment [18] was carried out on Ni metal using the scattering geometry in Fig. 2.

\subsection{Circular polarization}

Consider the vertical scattering geometry in Fig. 3 where the incident polarization is circular (complex, $\hat{\epsilon}=\hat{\sigma} \mp i \hat{\pi}$ ) and no assumptions are made about the direction of magnetization (beyond it being well defined). While magnetic scattering can take place in rotated $(\hat{\sigma}, \hat{\pi}) \rightarrow\left(\hat{\pi}^{\prime}, \hat{\sigma}^{\prime}\right)$ and unrotated $\left(\hat{\pi} \rightarrow \hat{\pi}^{\prime}\right)$ channels, and charge scattering leaves the polarization unrotated, their final polarization states overlap and hence non-vanishing $\mathrm{C}-\mathrm{M}$ interference scattering takes place. In this case, because of the complex character of $\hat{\epsilon}, \hat{\epsilon}^{\prime}$ and the multiple polarization channels, it is convenient to use the density matrix formalism $[19,20]$ in order to compute the scattering cross section. In this formalism, the total scattered intensity is proportional to $\operatorname{Tr}\left(\rho^{\prime}\right)$, where $\operatorname{Tr}=$ trace and $\rho^{\prime}$ is the density matrix of the scattered beam, given by $\rho^{\prime}=$ $f \rho f^{\dagger}$. Here $f$ (and its Hermitian conjugate $f^{\dagger}$ ) and $\rho$ are scattering amplitude (given in Eq. (1)) and density matrix of the incident beam, respectively, written in the $(\sigma, \pi)$ matrix representation where contributions to the cross section are separated 


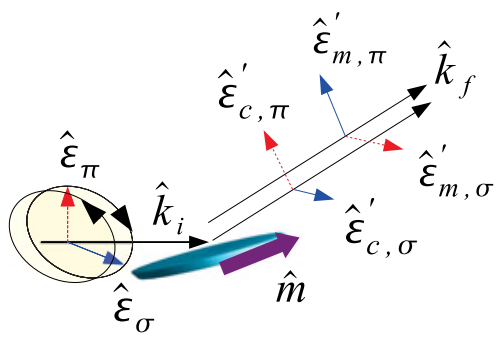

Fig. 3. Differential measurement of total scattered intensity for opposite X-ray helicities (without polarization analysis) yields C-M interference scattering sensitive to both direction and magnitude of magnetic moments in the scattering plane.

by the polarization channel to which they contribute, namely, $\left(\begin{array}{l}\sigma \rightarrow \sigma^{\prime} \pi \rightarrow \sigma^{\prime} \\ \sigma \rightarrow \pi^{\prime} \\ \pi \rightarrow \pi^{\prime}\end{array}\right)$ In this representation, the polarization terms in Eq. (1) are given by $\hat{\epsilon}_{c}^{\prime} \cdot \hat{\epsilon}=C=\left(\begin{array}{ll}1 & 0 \\ 0 & \hat{k}_{f} \cdot \hat{k}_{i}\end{array}\right)$ and $\left(\hat{\epsilon}_{m}^{\prime} \times \hat{\epsilon}\right) \cdot \hat{m}=M=\left(\begin{array}{cc}0 & -\hat{k}_{f} \cdot \hat{m} \\ \hat{k}_{i} \cdot \hat{m} & \left(\hat{k}_{f} \times \hat{k}_{i}\right) \cdot \hat{m}\end{array}\right)$, where a notation in terms of incident and outgoing unit wavevector $\hat{k}_{i}, \hat{k}_{f}$ is used for better contact with the experimental geometry $\left[\right.$ e.g. $\left(\hat{\pi}_{c}^{\prime} \cdot \hat{\pi}\right)=\left(\hat{k}_{f} \cdot \hat{k}_{i}\right)$ and $\left.\left(\hat{\sigma}_{m}^{\prime} \times \hat{\pi}\right) \cdot \hat{m}=\hat{k}_{i} \cdot \hat{m}\right][21]$. Note that in this derivation $\hat{\sigma}$ points into the plane of the paper, to be consistent with the notation of Blume and Gibbs [20]. In principle the contribution from the unrotated polarization in the magnetic scattering channel (diagonal term $\hat{\pi} \rightarrow \hat{\pi}^{\prime}$ ) yields sensitivity to the magnetization component perpendicular to the vertical scattering plane. However, as shown below, the contribution of this term to $\mathrm{C}-\mathrm{M}$ interference scattering vanishes in a differential measurement of scattered intensity for opposite X-ray helicities. As a result the $\mathrm{C}-\mathrm{M}$ interference scattering is sensitive to magnetization in the vertical scattering plane only, as derived below.

In order to evaluate $\operatorname{Tr}\left(f \rho f^{\dagger}\right)$ we use the density matrix $\rho$ for a circularly polarized incident X-ray beam [20]; $\rho=\frac{1}{2}\left(\begin{array}{cc}1 & i P_{c} \\ -i P_{c} & 1\end{array}\right)$ where $P_{c}= \pm 1$ corresponds to incident circular polarization with left and right helicity, respectively. With $C, M$ polarization matrices defined above, $f=f_{e} C-i f_{m} M$ (Eq. (1)) and the expression to evaluate is $\operatorname{Tr}\left[\left|f_{e}\right|^{2} C \rho C^{\dagger}+\left|f_{m}\right|^{2} M \rho M^{\dagger}+i f_{e} f_{m}^{*} C \rho M^{\dagger}-i f_{m} f_{e}^{*} M \rho C^{\dagger}\right]$; i.e., pure charge, pure magnetic, and $\mathrm{C}-\mathrm{M}$ interference scattering. Carrying out the matrix algebra, executing the trace operation (adding diagonal terms) and adding the structure factor contribution returns

$$
\begin{aligned}
I^{L, R}(Q, E)= & \frac{1}{2}\left[1+\cos ^{2} 2 \theta\right]\left|\sum_{n} f_{e, n}(Q, E) e^{i \boldsymbol{Q} \cdot \boldsymbol{r}_{n}}\right|^{2} \\
& +\left[\left(\hat{k}_{f} \cdot \hat{m}\right)^{2}+\left(\left(\hat{k}_{f} \times \hat{k}_{i}\right) \cdot \hat{m}\right)^{2}\right]\left|\sum_{n} f_{m, n}(E) e^{i \boldsymbol{Q} \cdot \boldsymbol{r}_{n}}\right|^{2} \\
& \pm \frac{\left|P_{c}\right|}{2}\left[\left(\hat{k}_{i} \cdot \hat{m}\right)+\left(\hat{k}_{f} \cdot \hat{m}\right) \cos 2 \theta\right]\left(\sum_{n, l} f_{e, n} f_{m, l}^{*} e^{i \boldsymbol{Q} \cdot\left(\boldsymbol{r}_{l}-\boldsymbol{r}_{n}\right)}+\text { c.c. }\right)
\end{aligned}
$$

where a collinear magnetic structure is assumed (i.e., $\hat{m}$-dependent polarization terms taken out of the summation in the structure factor calculation). Sensitivity to magnetization normal to the scattering plane is only obtained in the pure magnetic scattering channel, but this carries negligible intensity relative to the other terms (this may not be the case for stronger, soft X-ray resonances). A differential measurement of 


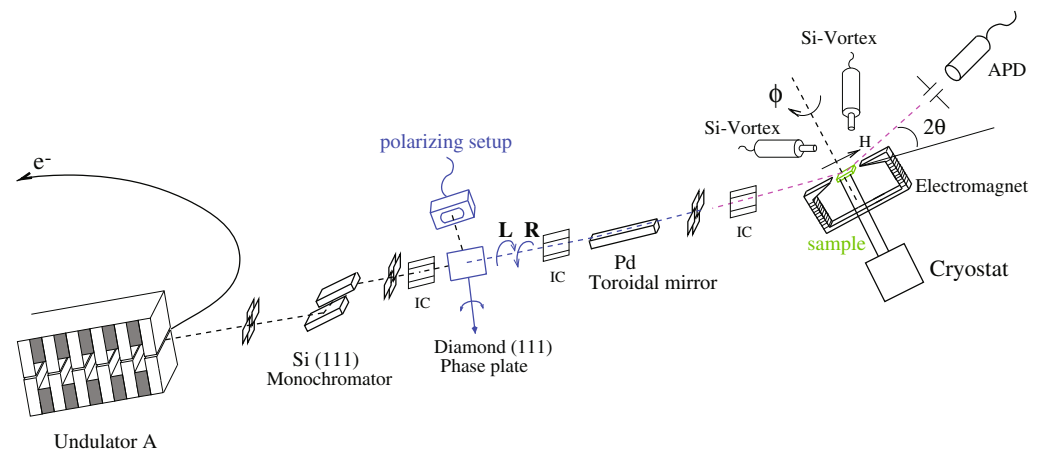

Fig. 4. Experimental setup for detection of C-M interference scattering at beamline 4-ID-D of the Advanced Photon Source.

scattered intensity for opposite $(\mathrm{L}, \mathrm{R}) \mathrm{X}$-ray helicity removes pure charge scattering and retains only the C-M interference term (twice the last term in Eq. (3)). This interference probes magnetization in the scattering plane only. A sum of scattered intensities, on the other hand, returns pure charge scattering (neglecting the pure magnetic scattering contribution). A flipping ratio is defined as

$$
\frac{I^{L}-I^{R}}{I^{L}+I^{R}}=\frac{P_{c}\left[\left(\hat{k}_{i} \cdot \hat{m}\right)+\left(\hat{k}_{f} \cdot \hat{m}\right) \cos 2 \theta\right]\left(\sum_{n, l} f_{e, n} f_{m, l}^{*} e^{i \boldsymbol{Q} \cdot\left(\boldsymbol{r}_{l}-\boldsymbol{r}_{n}\right)}+\text { c.c. }\right)}{\left[1+\cos ^{2} 2 \theta\right]\left|\sum_{n} f_{e, n}(Q, E) e^{i \boldsymbol{Q} \cdot \boldsymbol{r}_{n}}\right|^{2}} .
$$

The C-M interference scattering is sensitive to both the direction and magnitude of the element-specific magnetization, the former through the polarization dependent term, which yields sensitivity to the component of magnetization in the scattering plane, and the latter through the magnitude of the energy-dependent $f_{m}$. By exploiting structure factor effects in crystals and layered films (Q-dependence), site- and depth-specific magnetization can be retrieved, as discussed in Sect. 4.1 and 4.2. We now summarize some of the advantages of this detection method over the method described in Sect. 3.1. Fast helicity switching, such as enabled with phase retarding optics [22] or specialized insertion devices, allows quick consecutive measurements of scattered intensities for opposite helicities at each energy point in resonant scans at fix $\boldsymbol{Q}$, or at each $\boldsymbol{Q}$ point in $\boldsymbol{Q}$ scans at fix energy, hence reducing systematic errors associated with beam drifting or sample instabilities. (Slower magnetization switching, such as with a superconducting magnet, is not required for the measurement in this detection scheme.) Furthermore, since helicity switching and magnetization switching are redundant (both reverse the sign of C-M interference scattering), measurements with opposite direction of magnetization can be used to test the accuracy of the detected C-M interference signal (and to subtract artifacts in the data of nonmagnetic origin, if present). Additionally, since polarization switching is used in the measurement, the magnetic field dependence of $\mathrm{C}-\mathrm{M}$ interference scattering can also be studied.

\section{Experimental}

Experiments are carried out at undulator beamline 4-ID-D of the Advanced Photon Source, Argonne National Laboratory (Fig. 4). The beamline uses diamond C(111) Bragg transmission phase plates for converting the linearly polarized undulator beam into a circularly polarized beam with high degree of polarization $\left(P_{c} \gtrsim 0.95\right)[23]$. 
Fast helicity switching is achieved by offseting the phase plate in opposite directions away from the exact Bragg condition by means of a piezoelectric actuator. Since the transmissivity of phase plates differs for left and right helicities $(\approx 5 \%$ difference in intensity) [23], care must be taken to ensure that this asymmetry is properly normalized in measurements of C-M interference scattering. Since measurements are usually done on strong, allowed Bragg peaks (crystals) or strong specularly reflected beams (from films) a high count rate detector, such as an avalanche photodiode (APD, 5 nsec time resolution), is best suited for achieving the required signal-to-noise ratios (up to $\sim 10,000$ ) in reasonable times. (Counting times of a few-to-several seconds per energy or $\boldsymbol{Q}$ point at an undulator beamline are usually limited by detector dead time.) Note that the asymmetry in incident intensity for $\mathrm{L}, \mathrm{R}$ helicities can also introduce artificial differences in measured C-M interference intensity due to different dead time in the APD detector. Hence, the detector must be operated within its linear-response regime. It must also be ensured that any linear polarization component present in an imperfectly polarized circular beam is of equal magnitude for both $\mathrm{X}$-ray helicities. This is particularly important at high scattering angles where the pure charge scattering term is strongly dependent on the $\hat{\pi}$ polarization content of the X-ray beam.

Fluorescence detectors (Si-drift diode, or photodiodes) are used for simultaneous detection of X-ray absorption near edge structure (XANES) and X-ray magnetic circular dichroism (XMCD). The fluorescence data, which lacks site/depth specificity, is used to retrieve imaginary parts of resonant charge and magnetic scattering factors $\left(f_{c, m}^{\prime \prime}\right)$ while their real parts are obtained by means of a differential Kramers-Krönig (KK) transformation [24]. We detect C-M interference scattering by switching X-ray helicity at $1-2 \mathrm{~Hz}$ and measuring the incident and diffracted intensities for each X-ray helicity with a dual photon counter at each energy point in energy scans through a resonance, or at each $\boldsymbol{Q}$ point in $\boldsymbol{Q}$ scans at fixed resonant energy [25]. Usually about 20 helicity switches per $\mathrm{E} / \boldsymbol{Q}$ point are used in a measurement requiring high $\mathrm{S} / \mathrm{N}$ ratio. Large C-M interference signals ( $\gtrsim 1 \%$ ), which do not require extended time averaging, are simply measured by switching helicity once.

\subsection{Site-specific magnetism in a $\mathrm{Nd}_{2} \mathrm{Fe}_{14} \mathrm{~B}$ permanent magnet}

This example illustrates how the $\boldsymbol{Q}$-dependence (structure factor effects) and polarization dependence (sensitivity to magnetic moment direction) of C-M interference scattering can be exploited in ferromagnetic crystals to measure both element- and site-specific magnetism. $\mathrm{Nd}_{2} \mathrm{Fe}_{14} \mathrm{~B}$ has the highest energy product of all commercial permanent magnetic materials (a measure of both remanent magnetization and coercive field). The magnetic "hardness" of $\mathrm{Nd}_{2} \mathrm{Fe}_{14} \mathrm{~B}$, i.e., its resistance against demagnetizing fields (large coercivity) arises from the interaction between the anisotropic, partially filled $\mathrm{Nd} 4 f$ electronic shell with the crystal electric field (CEF) of surrounding charges, which reflects the symmetry of the crystalline environment [26]. The CEF dictates the orientation of the electronic orbitals relative to the crystalline axes, while a strong spin-orbit interaction within the $4 f$ levels forces the magnetic moment to follow suit. A peculiar feature of $\mathrm{Nd}_{2} \mathrm{Fe}_{14} \mathrm{~B}$ is the presence of two inequivalent $\mathrm{Nd}$ crystal sites in the crystal structure $(f$ and $g$ Wyckoff sites within the $\mathrm{P}_{2} / \mathrm{mnm}$ space group) [27]. While the absorption-based XMCD is element-specific and separates the magnetic contributions from $\mathrm{Nd}$ and $\mathrm{Fe}$ sites, it averages over the two inequivalent $\mathrm{Nd}$, and six inequivalent Fe, crystal sites. The contribution of each of the inequivalent sites to Bragg diffraction, however, depends on the structure factor and, hence, it is possible to vary these contributions by proper choice of 


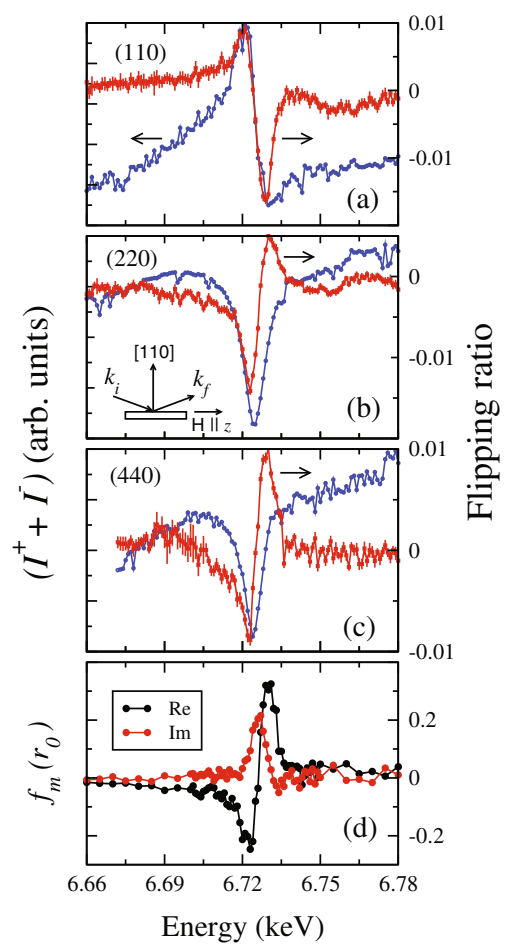

Fig. 5. Exploiting structure factor effects in a single crystal for element- and site-specific magnetism. Measurements under varying Bragg conditions selectively probe the magnetism of inequivalent $\mathrm{Nd}$ crystal sites.

scattering vector, $\boldsymbol{Q}$. For example, tuning to (110), (220) and (440) Bragg conditions probes Nd $g$ sites $(97 \%), \mathrm{Nd} f$ sites $(96.4 \%)$, or almost equal contributions from both, (48.5 and $51.5 \%$, respectively) [28-31]. A measurement of resonant C-M interference scattering under these special Bragg conditions yields element- and site-specific $\mathrm{Nd}$ magnetism.

We used a single crystal with the [110] surface oriented along the scattering vector in reflection geometry. Figure 5 shows energy scans through the $\mathrm{Nd} L_{2}$ edge while measuring the sum of scattered intensity for opposite X-ray helicity (resonant charge scattering, twice the first term in Eq. (3)) and the flipping ratio (Eq. (4)) under the three different fix $\boldsymbol{Q}$ conditions discussed above. The resonant magnetic scattering factor, $f_{m}$, obtained from fluorescence measurements and related KK transformation, i.e, averaged over inequivalent $\mathrm{Nd}$ sites, is also shown. During these measurements an $H=0.6 \mathrm{~T}$ magnetic field was applied along the easy-axis direction ( $\hat{c}$-axis) in the scattering plane in order to saturate the magnetization [28-31]. As discussed above, a singular advantage of detecting $\mathrm{C}-\mathrm{M}$ interference scattering with helicity switching, is the ability to check accuracy of $\mathrm{C}-\mathrm{M}$ interference signals by reversing the direction of the applied field, and to carry out field-dependent measurements. Data in Fig. 6 confirms the response of C-M interference scattering to a field reversal, as well as its insensitivity to magnetization normal to the scattering plane. We can exploit this polarization dependence to carry out measurements of site-specific magnetization reversal. Figure 6 also shows the field-dependence of C-M interference scattering (hysteresis loops) measured at (110) and (220) Bragg conditions and resonant energies that maximize the flipping ratio. The magnetic field is applied along the $\hat{c}$ easy-axis. 

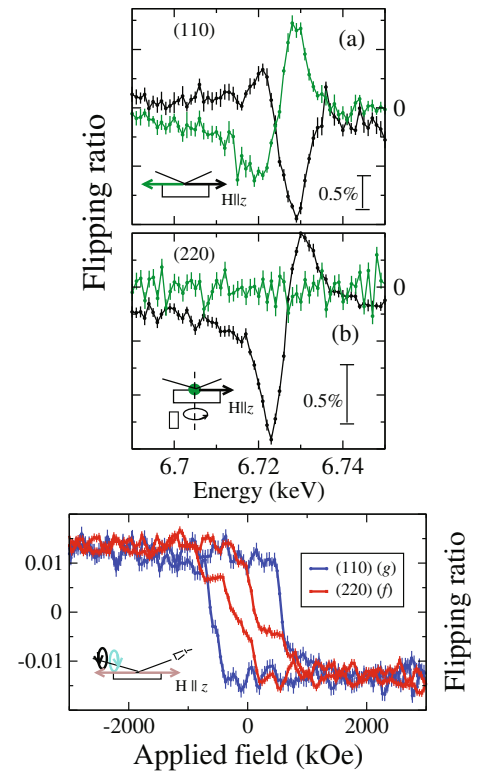

Fig. 6. (Top) angular dependence of C-M interference scattering. Magnetization reversal inverts the sign of $\mathrm{C}-\mathrm{M}$ interference. An applied field orthogonal to the scattering plane causes the C-M interference signal to vanish if the magnetization follows the applied field. Alternatively such transverse geometry can be used to probe for magnetization away from the applied field direction. (Bottom) element- and site-specific magnetization reversal in $\mathrm{Nd}_{2} \mathrm{Fe}_{14} \mathrm{~B}$. The $g$ sites strongly prefer a $c$-axis orientation, while the $f$ sites do not, indicating the $g$ sites are key in dictating the magneto-crystalline anisotropy of this permanent magnet [28-31].

(Note that a different choice of resonant energy would simply scale the hysteresis loop and possibly invert its sign, but would not change its field dependence). The $g$ sites display large coercivity and remanence while the $f$ sites display reduced remanence and marginal coercivity. In other words, the $g$ sites strongly prefer a $\hat{c}$-axis orientation (the easy-axis) while the $f$ sites do not have such a strong preference. This result, which was validated by calculations of site-specific magneto-crystalline anisotropy [28-31], indicate that $\mathrm{Nd} g$ sites dictate the magnetic "hardness" of $\mathrm{Nd}_{2} \mathrm{Fe}_{14} \mathrm{~B}$, a critical finding for guiding efforts to develop improved permanent magnets [28-31].

\subsection{Depth-resolved magnetization profiles in Gd/Fe multilayers}

The extension of the kinematic approach outlined in Sect. 3.2 for (imperfect) crystals, to the specular reflectivity channel in layered magnetic heterostructures is quite straightforward. The small $Q_{z}=\frac{4 \pi}{\lambda} \sin \theta<1 \AA^{-1}$ values attained in small-angle (longwavelength), specular reflectivity experiments in the hard (soft) X-rays renders the scattering insensitive to internal atomic structure $\left(Q_{z}^{-1}>\right.$ interatomic distance). Instead, the specular reflectivity is due to charge- and magnetic-density contrast at chemical and magnetic interfaces where changes in the (complex) index of refraction occur. When using circularly-polarized X-rays the expression for C-M interference scattering derived in Eqs. (3)-(4) can be mapped into the specular reflectivity channel [32-34] by replacing atoms with interfaces, atomic scattering factors with charge and magnetic density contrast, and crystallographic Debye-Waller factors (omitted 


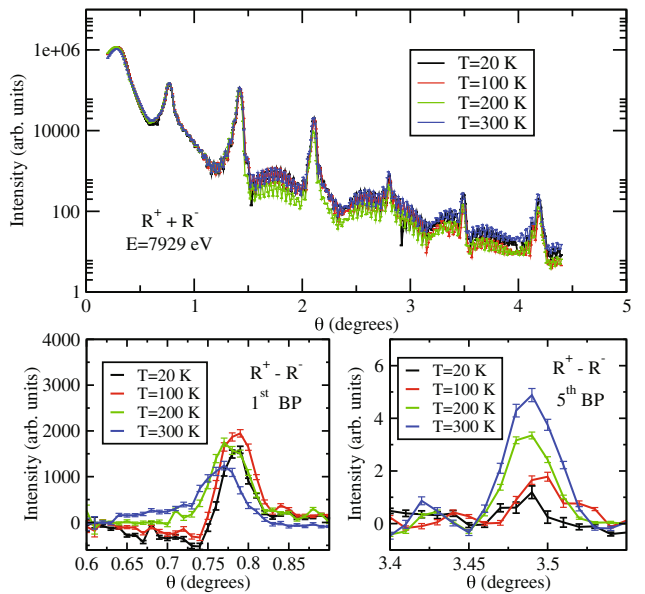

Fig. 7. Chemical (top) and C-M interference specular reflectivity in a $[\mathrm{Gd}(50 \AA) / \mathrm{Fe}(15 \AA)]_{15}$ multilayer measured at the $\operatorname{Gd} L_{2}$ edge.

in the previous equations) with interfacial roughness, to yield

$$
\begin{aligned}
& R^{L}-R^{R} \propto P_{c}\left[\left(\hat{k}_{i} \cdot \hat{m}\right)+\left(\hat{k}_{f} \cdot \hat{m}\right) \cos 2 \theta\right] \\
& \left(\sum_{k, l}^{N} \Delta \rho_{e, k} \Delta \rho_{m, l}^{*} e^{-Q_{z}^{2}\left(\sigma_{e, k}^{2}+\sigma_{m, l}^{2}\right) / 2} e^{i Q_{z}\left(z_{l}-z_{k}\right)}+c . c .\right),
\end{aligned}
$$

where the sumation is over all interfaces. In this expression $\Delta \rho_{e, k}=n_{e, k+1}\left(Z_{k+1}+\right.$ $\left.f_{c, k+1}\right)-n_{e, k}\left(Z_{k}+f_{c, k}\right)$ is the charge density contrast across interface $k, \Delta \rho_{m, l}^{*}=$ $n_{e, l+1} f_{m, l+1}^{*}-n_{e, l} f_{m, l}^{*}$ is the magnetic density contrast across interface $l, n_{e}$ is the atomic number density, $f_{c, m}$ resonant charge and magnetic scattering factors as before (note that the non-resonant charge form factor, $f_{0}(Q)$, is approximated by $\mathrm{Z}$ at low $\mathrm{Q}$ values), and $\sigma_{e, m}^{2}$ the charge and magnetic roughness, namely, mean-squares of height fluctuations about the average position of chemical and magnetic interfaces.

A full expression for the differential cross section can be found in Refs. [32-34]. Note that this kinematic approach (first Born approximation) is only valid in the weak scattering regime, i.e., away from the critical angle for total external reflection in the hard X-rays. A discussion of the limitation of this approach relative to the more accurate distorted wave Born approximation (DWBA) [35] is given in Ref. [36]. As before, scalar anomalous scattering factors are used corresponding to isotropic scattering factors (independent of polarization direction).

We illustrate the technique with results on a $\mathrm{Gd} / \mathrm{Fe}$ multilayered model system. A strong antiferromagnetic coupling between Gd and Fe magnetic moments at the $\mathrm{Gd} / \mathrm{Fe}$ interface, together with quite different ferromagnetic ordering temperatures $\mathrm{T}_{\mathrm{c}}$ for $\mathrm{Fe}(1,024 \mathrm{~K})$ and $\mathrm{Gd}(293 \mathrm{~K})$, makes this an interesting ferri-magnetic system $[37,38]$. Its net magnetization can be tuned to zero (compensation), and complex, collinear/twisted magnetic states can be prepared, by tuning magnetic field and temperature $[39,40]$. A magnetization that varies in magnitude and/or direction across the multilayer's depth is characteristic of these states.

Figure 7 shows resonant charge reflectivity (sum of reflected intensity for L and $\mathrm{R}$ helicities) and $\mathrm{C}-\mathrm{M}$ interference reflectivity (difference of reflected intensities) as a function of temperature for a polycrystalline $[\operatorname{Gd}(50 \AA) / \mathrm{Fe}(15 \AA)]_{15}$ multilayer collected near the $\mathrm{Gd} L_{2}$ edge $(E=7,929 \mathrm{eV})$. While the chemical (charge) reflectivity is $\mathrm{T}$-independent, the $\mathrm{C}-\mathrm{M}$ interference signal shows strong T-dependence due to the 

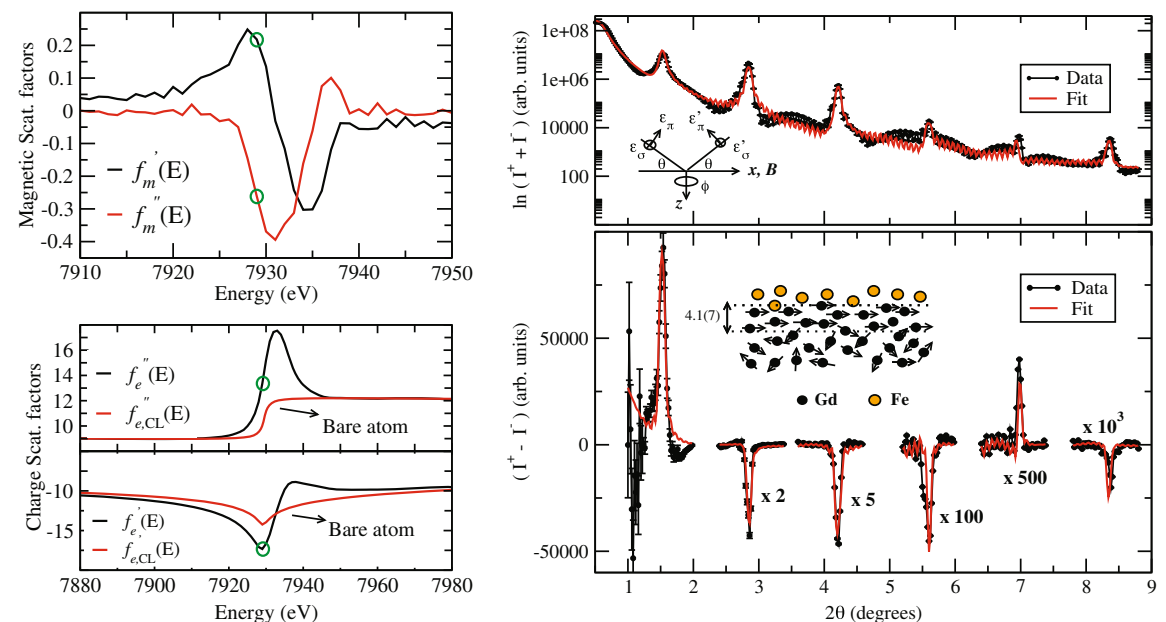

Fig. 8. (Left) anomalous scattering factors at the $\mathrm{Gd} L_{2}$ edge determined from XANES/XMCD measurements and a differential Kramers-Krönig transformation using Cromer-Liberman (CL) tabulated cross sections away from resonance. (Right) charge (top) and $\mathrm{C}-\mathrm{M}$ interference specular reflectivity at $\mathrm{T}=300 \mathrm{~K}$, together with corresponding fits. The presence of a polarized Gd interfacial region near Fe is deduced from the fits [34].

demagnetization of Gd in the interior of (low $\mathrm{T}_{\mathrm{c}}$ ) Gd layers. However, strong C-M interference signal remains present even at $T=300 \mathrm{~K}$, which is above the ordering temperature of bulk Gd. Quantitative fits (Fig. 8) using the expression in Eq. (5) and resonant scattering factors determined experimentally from XANES/XMCD measurements of depth-averaged absorption coefficients (Fig. 8) show that Gd regions near the Gd/Fe interface, about $4 \AA$-thick, remain fully magnetized at room temperature due to proximity to the high $\mathrm{T}_{\mathrm{c}}$ Fe layers [34]. The extreme sensitivity and high spatial resolution of $\mathrm{C}-\mathrm{M}$ interference scattering is therefore an asset for studies of the interfacial magnetism in ferro/ferri-magnetic systems of technological relevance such as exchange-bias, giant/tunnel magnetoresistance, and other spintronic devices.

Note that at low $\mathrm{T}$, where the Gd layers are uniformly magnetized, the chemical and magnetic interfaces coincide so the sign of the C-M interference remains the same at different multilayer Bragg peaks [36]. At $\mathrm{T}=300 \mathrm{~K}$, however, the additional purely magnetic interface $4.1 \AA$ away from the Gd/Fe interface strongly affects the Q-dependence of charge-magnetic interference, the sign of which alternates between multilayer Bragg peaks. The application of C-M interference scattering for the determination of non-collinear magnetic structures (Fig. 9) was recently demonstrated [7], where intra-layer twisted structure was refined with near atomic resolution (polarization dependent term in Eq. (5) becomes depth-specific; i.e., moves inside the summation). We have also recently shown the potential benefit of simultaneous refinements of $\mathrm{C}-\mathrm{M}$ interference scattering and polarized neutron reflectometry data for more robust modeling of magnetization depth profiles in artificial layered nanostructures [7].

\section{Summary}

We have outlined the relevant interactions, and necessary conditions, leading to charge-magnetic interference scattering in ferro(ferri)-magnetic materials where charge and magnetic scattering overlap in reciprocal space. Different routes for detecting C-M interference scattering were presented, the advantages and limitations of 

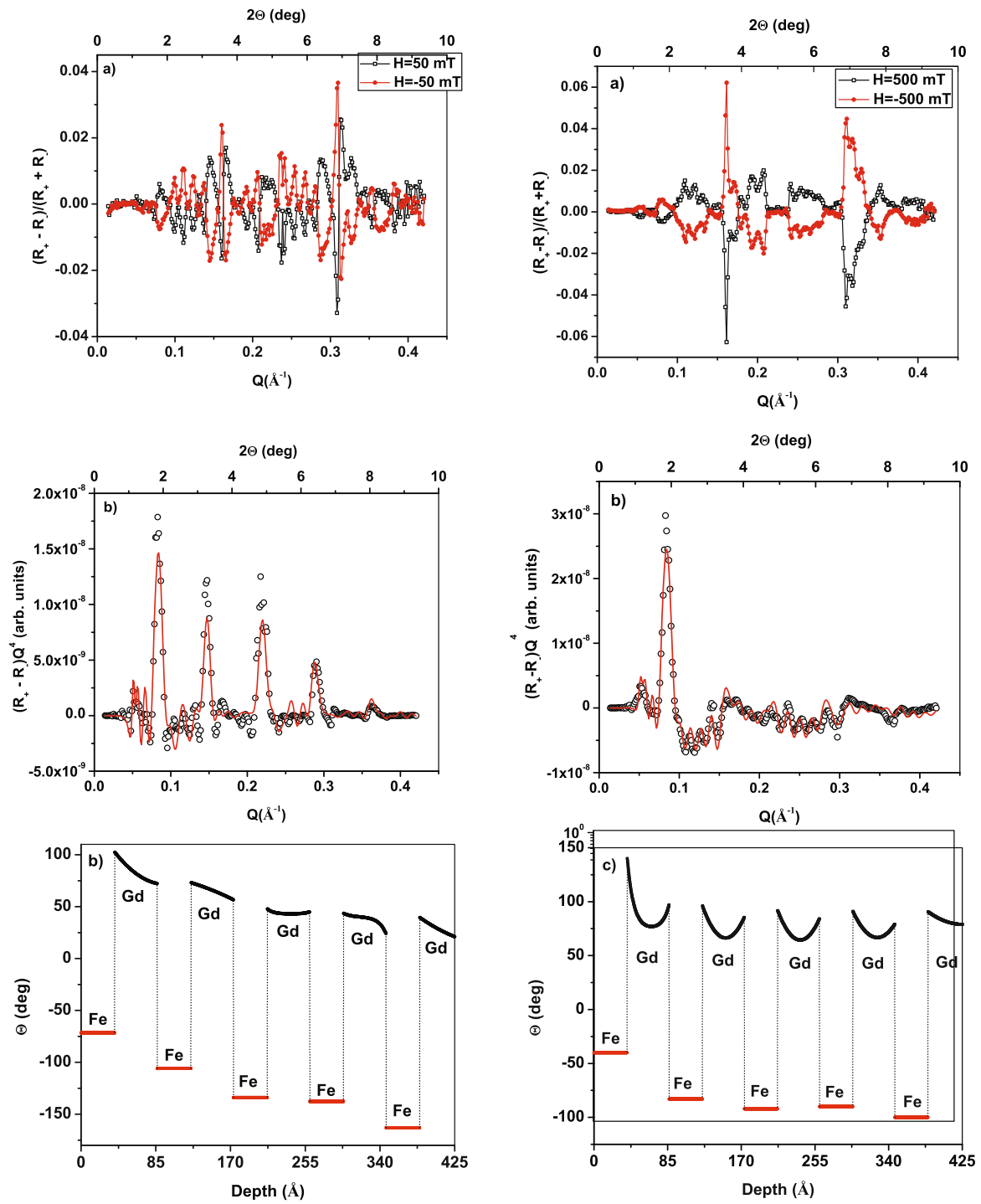

Fig. 9. C-M interference scattering data (top panel), and fits (middle panel), for an $[\mathrm{Fe}(35 \AA) / \mathrm{Gd}(50 \AA)]_{5}$ layered structure at $\mathrm{T}=20 \mathrm{~K}$ and $\mathrm{H}=0.5 \mathrm{kOe}$ (left) and $\mathrm{H}=5 \mathrm{kOe}$ (right) applied fields. A surface-twisted state is observed at both fields, characterized by a stronger deviation of magnetization density away from the applied field direction near the top surface compared to the bottom of the multilayer (bottom panel). An antiparallel alignment of $\mathrm{Fe}$ and $\mathrm{Gd}$ moments is maintained at the $\mathrm{Gd} / \mathrm{Fe}$ interfaces. At higher fields (right panel) the Gd moments are strongly inhomogeneous within the layer as the interfacial coupling forces the Gd moments against the field, while minimization of Zeeman energy forces the moments in the interior of the Gd layers towards the field. A weak intralayer Gd exchange coupling allows this twist to take place [7].

each discussed in detail. The ability of this probe to yield site- and depth-specific magnetization in crystals and layered nanostructures, respectively, was illustrated with examples from our previous work on bulk permanent magnet $\mathrm{Nd}_{2} \mathrm{Fe}_{14} \mathrm{~B}$, and artificial Gd/Fe multilayer model systems. The development of fast polarization-switching capabilities based on phase-retarding optics or specialized insertion devices, coupled 
with novel detection schemes, will continue to enhance the potential of C-M interference scattering experiments for a better understanding of complex ferro(ferri)magnetic materials and nanostructures.

Work at Argonne and Ames is supported by the U.S. Department of Energy, Office of Science, under contract Nos. DE-AC02-06CH11357 and DE-AC02-07CH11358, respectively. We thank S. Sinha, D. R. Lee, M. van Veenendaal, H. Hashizume, H. Hosoito, R. Camley. J. Meersschaut, Christie Nelson, A. Ankudinov, J. Pollmann, A. Cady, G. Subias, J. Garcia, E. Lorenzo, J. Cross, M. Newville, S. te Velthuis and B. Kirby for valuable input in theoretical, experimental and modeling aspects of this work.

\section{References}

1. J.P. Hannon, G.T. Trammell, M. Blume, D. Gibbs, Phys. Rev. Lett. 61, 1245 (1988)

2. P. Carra, M. Altarelli, F. de Bergevin, Phys. Rev. B 40, 7325 (1989)

3. D. Gibbs, G. Grübel, D.R. Harshman, E.D. Isaacs, D.B. McWhan, D. Mills, C. Vettier, Phys. Rev. B 43, 5663 (1991)

4. F. de Bergevin, M. Brunel, Acta Cryst. A37, 314 (1981)

5. P.M. Platzman, N. Tzoar, Phys. Rev. B 2, 3556 (1970)

6. M. Blume, J. Appl. Phys. 57, 3615 (1985)

7. E. Kravtsov, D. Haskel, S.G. E te Velthuis, J.S. Jiang, B.J. Kirby, Phys. Rev. B 79, $134438(2009)$

8. D. Gibbs, G. Gübel, D.R. Harshman, E.D. Isaacs, D.B. McWhan, D. Mills, C. Vettier, Phys. Rev. B 43, 5663 (1991)

9. M. Elzo, E. Jal, O. Bunau, S. Grenier, Y. Joly, A.Y. Ramos, H.C.N. Tolentino, J.M. Tonnerre, N. Jaouen, J. Magn. Magn. Mater. 324, 105 (2012)

10. M. Sacchi, C.F. Hague, L. Pasquali, A. Mirone, J.-M. Mariot, P. Isber, E.M. Gullikson, J.H. Underwood, Phys. Rev. Lett. 81, 1521 (1998)

11. E.D. Isaacs, et al., Phys. Rev. Lett. 62, 1671 (1989)

12. D. McWhan, et al., Phys. Rev. B 42, 6007 (1990)

13. W.G. Stirling, G.H. Lander, Synchrotron Radiation News 5, 17 (1992)

14. S. Langridge, et al., Europhysics Lett. 25, 137 (1994)

15. P. Carra, M. Altarelli, F. de Bergevin, Phys. Rev. B 40, 7324 (1989)

16. A. Fasolino, P. Carra, M. Altarelli, Phys. Rev. B 47, 3877 (1993)

17. S.D. Brown, P. Strange, L. Bouchenoire, B. Zarychta, P.B.J. Thompson, D. Mannix, S.J. Stockton, M. Horne, E. Arola, H. Ebert, Z. Szotek, W.M. Temmerman, D. Fort, Phys. Rev. Lett. 99, 247401 (2007)

18. K. Namikawa, M. Ando, T. Nakajima, H. Kawata, J. Phys. Soc. Jpn. 54, 4099 (1985)

19. U. Fano, Rev. Mod. Phys. 29, 74 (1957)

20. M. Blume, D. Gibbs, Phys. Rev. B 37, 1779 (1988)

21. J.P. Hill, D.F. McMorrow, Acta Cryst. A52, 236 (1996)

22. K. Hirano, K. Izumi, T. Ishikawa, S. Annaka, S. Kikuta, Jpn. J. Appl. Phys. 30, L407 (1991)

23. J.C. Lang, G. Srajer, Rev. Sci. Instrum. 66, 1540 (1995)

24. J.O. Cross, M. Newville, J.J. Rehr, L.B. Sorensen, C.E. Bouldin, G. Watson, T. Gouder, G.H. Lander, M.I. Bell, Phys. Rev. B 58, 11215 (1998)

25. Y. Hayasaki, K. Ishiji, H. Hashizume, H. Hosoito, K. Homote, M. Kuribayashi, G. Srajer, J. Lang, D. Haskel, J. Phys. Cond. Matter 16, 1915 (2004)

26. J.F. Herbst, Rev. Mod. Phys. 63, 819 (1991)

27. J.F. Herbst, J.J. Croat, F.E. Pinkerton, W.B. Yelon, Phys. Rev. B 29, 4176 (1984)

28. D. Haskel, J.C. Lang, Z. Islam, A. Cady, G. Srajer, M. van Veenendaal, P.C. Canfield, Phys. Rev. Lett. 95, 217207 (2005)

29. A. Koizumi, K. Namikawa, H. Maruyama, K. Mori, H. Yamazaki, Jpn. J. Appl. Phys. 32, Suppl. 32-2, 332 (1993) 
30. F. Bartolome, J.M. Tonnerre, N. Jaouen, D. Raoux, J. Chaboy, L.M. Garcia, H. Maruyama, R. Steinmann, J. Appl. Phys. 87, 4762 (2000)

31. D. Haskel, M. van Veenendaal, Phys. Rev. Lett. 97, 259702 (2006)

32. S.K. Sinha, et al., Phys. Rev. B 38, 2297 (1988)

33. R.M. Osgood III, et al., J. Magn. Magn. Mater. 198-199, 698 (1999)

34. D. Haskel, G. Srajer, J.C. Lang, J. Pollmann, C.S. Nelson, J.S. Jiang, S.D. Bader, Phys. Rev. Lett. 87, 207201 (2001)

35. D.R. Lee, S.K. Sinha, D. Haskel, Y. Choi, J.C. Lang, S.A. Stepanov, G. Srajer, Phys. Rev. B 68, 224409 (2003)

36. Y. Choi, D. Haskel, R. Camley, D.R. Lee, J. Lang, G. Srajer, J. Jiang, S.D. Bader, Phys. Rev. B 70, 134420 (2004)

37. R.E. Camley, D.R. Tilley, Phys. Rev. B 37, 3413 (1988)

38. R.E. Camley, Phys. Rev. B 39, 12316 (1989)

39. J.G. LePage, R.E. Camley, Phys. Rev. Lett. 65, 1152 (1990)

40. R.E. Camley, Phys. Rev. B 35, 3608 (1987) 